\title{
28 Research Square \\ Is Tracheostomy Safe?: Common Indications and Early Complications Among Ugandan Patients In A Pre-Covid-19 Era.
}

Daniel James Nyanzi ( $\nabla$ nyanzidaniel4@gmail.com )

Kabale University School of Medicine https://orcid.org/0000-0001-8990-9277

Daniel Atwine

Mbarara University of Science and Technology Faculty of Medicine

Ronald Kamoga

Mbarara University of Science and Technology Faculty of Medicine

Caroline Birungi

SOAR research foundation

Caroline A Nansubuga

Makerere University Medical School: Makerere University College of Health Sciences

Victoria Nyaiteera

Mbarara University of Science and Technology Faculty of Medicine

Doreen Nakku

Mbarara University of Science and Technology Faculty of Medicine

\section{Research}

Keywords: Tracheostomy, Indication, Incidence, Early complications

Posted Date: October 29th, 2021

DOl: https://doi.org/10.21203/rs.3.rs-963625/v1

License: (c) (i) This work is licensed under a Creative Commons Attribution 4.0 International License.

Read Full License 


\section{Abstract}

Background: Tracheostomy is a life-saving procedure whose outcomes may vary between hospitals based on disparities in their existing expertise. We aimed at establishing the indications, early tracheostomy-related complications and their associated factors in Uganda.

Methods: In a prospective cohort study, we consecutively enrolled one-hundred patients, both adults and children 2 hours post-tracheostomy procedure. At baseline, information on patients' socio-demographics, tracheostomy indications, pre- and post-procedural characteristics was collected through researcher administered questionnaires and from medical records. Clinical examination was performed at baseline but also at either day 7 or whenever a tracheostomy-related complication was suspected during the 7days follow-up. Comparison of patients' baseline characteristics, tracheostomy indications and complications across two hospitals was done using Pearson's chi-square. For predictors of early tracheostomy complications, bivariate and multivariate analysis models were fitted using binomial regression in STATA 13.0 software.

Results: Majority of patients were adults (84\%) and males (70\%). The commonest tracheostomy indications were; pulmonary toilet (58\%) and anticipated prolonged intubation (42\%). Overall, 53\% (95\% Cl: 43.0 - 62.7) had early complications with the commonest being tube obstruction (52.6\%). Independent predictors of early tracheostomy-related complications were; anticipated having prolonged intubation as an indication ( $\mathrm{RR}=1.8,95 \% \mathrm{Cl}: 1.19-2.76)$, Bjork flap tracheal incision ( $\mathrm{RR}=1.6,95 \% \mathrm{Cl}: 1.09$ - 2.43), vertical tracheal incision ( $R R=1.53,95 \% \mathrm{Cl}$ : $1.02-2.27)$, and age below 18 years $(R R=1.22$, $95 \% \mathrm{Cl}: 1.00-1.47)$.

Conclusion: Within a pre-COVID-19 context, pulmonary toilet is the commonest tracheostomy indication at major hospitals in Uganda. The incidence of early tracheostomy complications is high and majorly related to post-procedure tracheostomy tube management. Having anticipated prolonged intubation as an indication for tracheostomy, a Bjork flap or vertical tracheal incisions and being a child increases the risk of complications. Emphasis on surgical training and post-procedural management for both clinicians and nurses should be made. New data will be needed within the COVID-19 era.

\section{Background}

Tracheostomy is a life-saving surgical procedure used to secure the airway. There are a number of indications for tracheostomy but the common ones include acute respiratory failure, anticipated need for prolonged mechanical intubation, broncho-pulmonary toilet, and impacted airway foreign bodies among others (Johnson, Rosen et al. 2014, Abe, Madotto et al. 2018). In the intensive care unit (ICU), the procedure has been noted to reduce the requirements for patient sedation when prolonged intubation is anticipated, improve patient comfort especially if ventilated, and facilitate earlier resumption of patient respiratory autonomy (Barry and Bodenham 2004, Abe, Madotto et al. 2018). 
The many benefits of tracheostomy have led to a global upward trend in the frequency of the procedure with up-to 250,000 procedures currently performed annually in resource-rich countries (Brenner, Pandian et al. 2020) and indications are being continuously revised. In literature, this rising trend has been attributed to increased access to better intensive care services (Kawale, Keche et al. 2017) and changes in the epidemiology of upper airway obstructive conditions (Adetinuola, Amusa et al. 2011). In the past, obstructive airway disease secondary to acute aero-digestive infections was the most common indication, but in the recent pre-COVID-19 years, trauma and aero-digestive tumors have dominated the list (Adetinuola, Amusa et al. 2011, Gilyoma, Balumuka et al. 2011, Cheung and Napolitano 2014).

Like all surgical procedures, tracheostomy carries a risk of adverse events that may increase patient morbidity, prolong hospital stay and add undue strain to an already low resourced healthcare system (Gilyoma, Balumuka et al. 2011, Abe, Madotto et al. 2018, Cramer, Graboyes et al. 2019). Common complications of tracheostomy include stomal site infection, bleeding, tube obstruction and inadvertent tube dislodgement among others. Even though literature suggests that tracheostomy related complication rates range from 6 to $66 \%$ with a mortality rate of up-to $60 \%$ depending on existing comorbidities (Hamid, Sattar et al. 2011, Halum, Ting et al. 2012, Brenner, Pandian et al. 2020), it is unclear if this can be generalized to resource limited settings. Furthermore, differences in hospital resources and policies may play a contributory role to variation in patient outcomes and complication rates. For example, in high resource centers, different cadre of staff ranging from surgical residents, anesthesiologists, surgeons and critical care nurses may be trained to perform this complex procedure and care for the patients thereafter. However, in low resource settings like Uganda, it is a reserve for specially trained and in-training medical personnel such as otolaryngologists and oromaxillofacial surgeons while post-procedural care is offered by the nurses and patient attendants. We hypothesize that this may contribute to variation in complication rates for tracheostomy. Other factors that have been documented in literature to be associated with tracheostomy-related complications include; obesity, patient age and tracheostomy type among others (Gilyoma, Balumuka et al. 2011, Karuga, Oburra et al. 2012, Nyansikera and Kirui 2013, Taylor and Randal 2015). Unfortunately, there is scanty literature on the incidence of tracheostomy-related complications in Uganda.

Therefore, our study aimed at establishing the indications for tracheostomy, the incidence of early tracheostomy-related complications and their associated factors in Uganda.

\section{Methods}

We conducted an observational prospective cohort study of adult and child patients who had under-gone tracheostomy at least 2 hours prior to recruitment. A patient was excluded from participation if he or she had: a history of a known bleeding disorder, had no caretaker in hospital, and if they had had the tracheostomy performed from other hospitals besides those involved in this study. In order to achieve the sample size, recruitment was performed at two large university training hospitals in Uganda, that is, Mbarara Regional Referral Hospital (MRRH) in South western Uganda, and Mulago National Referral 
Hospital (MNRH) in the capital city of Uganda. In both hospitals, the Otolaryngology division is solely tasked with performing tracheotomy procedures.

The sample size of 100 patients was calculated using the formula for estimation of a single population proportion, that is; $n=Z^{2 *} P(1-P) / r^{2}$ (Kelsey, Whittemore et al. 1996), where: $Z$ = standard normal deviation for two-tailed test based on alpha level (relates to the confidence interval level), assumed at $95 \%=1.96 ; \mathrm{P}$ is the proportion of patients with tracheostomy-related complications, assumed to be 0.42 , based on a $42 \%$ prevalence of tracheostomy related complications reported in a study in Kenya (Karuga, Oburra et al. 2012); $r$ is the margin error of estimation that was assumed to be 0.1 , that is $10 \%$, plus a $6 \%$ addition in sample size to cater for attrition. Ethical clearance was received from the MUST-IREC (MUSTREC 01/11-17) prior to commencement of study activities.

Written informed consent of eligible patients was obtained prior to enrolment into the study. At both sites, patients were consecutively recruited basing on the eligibility criteria two hours post-procedure from different units likely to host tracheotomy patients including the intensive care and high dependence units, emergency units and surgical wards. The 2-hour period was anticipated to be adequate for a patient's post-anesthetic stability and safe transfer from the procedure room to the host ward for post-operative monitoring and care. The decision was further guided by reports that tube obstruction, which is the most anticipated early complication is more likely to occur after 2 hours post-procedure (Nyansikera and Kirui 2013). For patients who were not fully conscious at the time of enrolment, consent was sought from their attendants.

Patients' socio-demographics, indications of tracheostomy, pre- and post-procedural factors including complications were collected at baseline from the patients, their medical records and caretakers using a researcher administered questionnaire. A baseline and day 7 clinical examination was performed so as to establish the complications of tracheostomy. In addition, clinical examination was performed whenever a complication was suspected during the 7 days of follow-up. As a quality control measure, the questionnaire was pretested before its use in the study. The principal investigator also trained the research assistants who were ICU nurses and otolaryngology residents in the study procedures and especially how to recognize the tracheostomy-related complications.

Data was analyzed using STATA version 13.0. Patients' baseline characteristics were described using medians for continuous variables and proportions for categorical variables. Stratified analysis was conducted to compare the frequency of each indication across hospitals using Pearson chi-square test. Complications were reported as frequencies and a composite variable of tracheostomy-related complication was generated as a binary variable coded $0=$ "No early tracheostomy-related complication", and 1 = "early Tracheostomy-related complication". An individual was considered to have an early tracheostomy-related complication if he/she had at-least one of the pre-defined complications both at enrolment and over the 7 days of study follow up. The overall proportion of patients with at-least one early tracheostomy related complication was calculated as the number of patients who developed at least one complication out of the total number of patients enrolled and expressed as a percentage, while 
the incidences of specific complications were calculated as frequencies of specific complications out of the total cumulative number of complication events and expressed as percentages. For complications such as tube obstruction which occurred more than once in some patients, only one event was recorded per patient and the total number of reported such events for all patients constituted the total cumulative number of complications.

Using chi-square and binomial regression models in univariate and multivariate analysis, the factors associated with early tracheostomy-related complications during follow-up were established. A significance level of $5 \%$ was used. Both unadjusted and adjusted risk-ratios were presented in tables with their corresponding 95\% Confidence Intervals.

\section{Results}

A total of 100 patients who had undergone tracheostomy were recruited into the study over a period of one year from MNRH and MRRH. Majority of these were male (70\%) and adult patients (84\%). Majority of the tracheostomies were performed in ICU/HDU settings (54\%), these were emergency tracheostomies in $43 \%$ of patients. Peri-operative antibiotics were administered in $60 \%$ of patients. Table 1 
Table 1

Sociodemographic and peri-surgical characteristics of patients, overall and by Hospital stratification

\section{Characteristic}

Gender

Male

Female

Age Categories (Years)

Adults $(\geq 18)$

Pediatric $(<18)$

Family Socioeconomic class*

Upper middle

Lower middle

Upper lower

Overall, $\mathrm{N}=100, \mathrm{n}$

(\%)

70 (70.0)

$30(30.0)$

84 (84.0)

$16(16.0)$

$7(7.1)$

$23(23.5)$

$54(54.1)$

14 (14.3)

Lower

Admission diagnosis

TBI/stroke

Upper airway obstruction

Others

Site for surgery

Emergency/Casualty

ICU/HDU

Operating theatre

Wards (ENT/OMF)
$\mathrm{MNRH}, \mathrm{N}=50, \mathrm{n}$

(\%)

$33(66.0)$

$17(34.0)$

39 (78.0)

$11(22.0)$

$45(90.0)$

0.102

$5(10.0)$
MRRH, N=50, n

(\%)

pvalue

37 (74.0)

0.383

13 (26.0) 


\begin{tabular}{|c|c|c|c|c|}
\hline Characteristic & $\begin{array}{l}\text { Overall, } \mathrm{N}=100, \mathrm{n} \\
(\%)\end{array}$ & $\begin{array}{l}\text { MNRH, N=50, n } \\
(\%)\end{array}$ & $\begin{array}{l}\text { MRRH, N=50, n } \\
(\%)\end{array}$ & $\begin{array}{l}\mathrm{p}- \\
\text { value }\end{array}$ \\
\hline Type of tracheostomy & $57(57.0)$ & $25(50.0)$ & $32(64.0)$ & 0.157 \\
\hline Elective & $43(43.0)$ & $25(50.0)$ & $18(36.0)$ & \\
\hline \multicolumn{5}{|l|}{ Emergency } \\
\hline Type of tracheal incision & $39(39.0)$ & $2(4.0)$ & $37(74.0)$ & \multirow[t]{5}{*}{0.000} \\
\hline T-incision & $9(9.0)$ & $0(0.0)$ & $9(18.0)$ & \\
\hline Bjork flap & $48(48.0)$ & $47(94.0)$ & $1(2.0)$ & \\
\hline Vertical & $4(4.0)$ & $1(2.0)$ & $3(6.0)$ & \\
\hline \multicolumn{4}{|l|}{ Horizontal } & \\
\hline $\begin{array}{l}\text { Closure of skin surgical } \\
\text { incision* }\end{array}$ & $71(71.7)$ & $25(50.0)$ & $46(94.0)$ & 0.000 \\
\hline $\begin{array}{l}\text { Cuffed tracheostomy tube } \\
\text { use }\end{array}$ & $89(89.0)$ & $47(94.0)$ & $42(84.0)$ & 0.110 \\
\hline $\begin{array}{l}\text { Disposable Internal Cannula } \\
\text { use }\end{array}$ & $28(28.0)$ & $3(6.0)$ & $25(50.0)$ & 0.000 \\
\hline Peri-operative Antibiotic use & $60(60.0)$ & $24(48.0)$ & $36(72.0)$ & 0.014 \\
\hline \multicolumn{5}{|c|}{$\begin{array}{l}\text { Family socioeconomic classification based on the } 2018 \text { modified Kuppuswamy Socioeconomic Scale } \\
\text { (Saleem 2018) }\end{array}$} \\
\hline \multicolumn{5}{|c|}{$\begin{array}{l}\text { *2 patients had missing data for socioeconomic class, }{ }^{*} \text { One patient had missing data for closure of } \\
\text { skin surgical incision. Other admission diagnosis included; Abdominal trauma, cut throat injury, } \\
\text { lingual cancer, and temporomandibular joint ankylosis. }\end{array}$} \\
\hline
\end{tabular}

\section{Indications for tracheostomy}

Multiple indications for tracheostomy were noted in $69 \%$ of patients. The most common indications are; pulmonary toilet (58\%), anticipated prolonged intubation (42\%), upper airway obstruction (36\%) and airway protection (28\%). There were no significant disparities in all indications across hospitals except for airway protection which was more commonly encountered in patients at MNRH (40\%) as compared MRRH (16\%), $p=0.008$. Table 2 
Table 2

Indications for tracheostomy, overall and by hospital stratification

\begin{tabular}{|lllll|}
\hline Indication for tracheostomy & $\begin{array}{l}\text { Overall, } \mathbf{N}=\mathbf{1 0 0}, \mathbf{n} \\
(\%)\end{array}$ & $\begin{array}{l}\text { MNRH, } \mathbf{N}=\mathbf{5 0 ,} \mathbf{n} \\
(\%)\end{array}$ & $\begin{array}{l}\text { MRRH, } \mathbf{N}=\mathbf{5 0}, \mathbf{n} \\
(\%)\end{array}$ & $\begin{array}{l}\text { p- } \\
\text { value }\end{array}$ \\
\hline Pulmonary toilet & $58(58.0)$ & $31(62.0)$ & $27(54.0)$ & 0.418 \\
\hline $\begin{array}{l}\text { Anticipated prolonged } \\
\text { intubation }\end{array}$ & $42(42.0)$ & $18(36.0)$ & $24(48.0)$ & 0.224 \\
\hline Upper airway obstruction & $36(36.0)$ & $20(40.0)$ & $16(32.0)$ & 0.405 \\
\hline Airway protection & $28(28.0)$ & $20(40.0)$ & $8(16.0)$ & 0.008 \\
\hline $\begin{array}{l}\text { Anticipated difficult } \\
\text { intubation }\end{array}$ & $9(9.0)$ & $3(6.0)$ & $6(12.0)$ & 0.295 \\
\hline $\begin{array}{l}\text { Adjunct to head and neck } \\
\text { surgery }\end{array}$ & $4(4.0)$ & $3(6.0)$ & $1(2.0)$ & 0.119 \\
\hline $\begin{array}{l}\text { Failed intubation } \\
\text { Failed extubation }\end{array}$ & $2(2.0)$ & $1(2.0)$ & $1(2.0)$ & 1.000 \\
\hline $\begin{array}{l}\text { Note: } \mathrm{N}=\text { total number of patients, } \mathrm{n}=\text { number of patients who had particular indications, some had } \\
\text { multiple indications }\end{array}$ & $1(1.0)$ & 0.591 \\
\hline
\end{tabular}

Table 3 shows that upper-airway obstruction was the commonest indication among males (93.7\%) while airway protection was the commonest among females (62.5\%) at $\mathrm{MRRH}$, and this difference was statistically significant, $p<0.05$. There were also no major disparities in indications of tracheostomy between adult and child patients in MRRH. Overall, at MNRH, no significant disparities were noted in indications for tracheostomy across gender and age categories. 
Table 3

Indications of tracheostomy stratified by age and gender and their variation between hospitals

\begin{tabular}{|c|c|c|c|c|c|c|c|c|}
\hline \multirow[t]{3}{*}{ Variable } & \multicolumn{8}{|c|}{ Indication } \\
\hline & \multicolumn{2}{|c|}{$\begin{array}{l}\text { Pulmonary } \\
\text { toilet: } \mathrm{N}=58\end{array}$} & \multicolumn{2}{|c|}{$\begin{array}{l}\text { Anticipated prolonged } \\
\text { intubation: } N=42\end{array}$} & \multicolumn{2}{|c|}{$\begin{array}{l}\text { Upper airway } \\
\text { obstruction: } \mathrm{N}=36\end{array}$} & \multicolumn{2}{|c|}{$\begin{array}{l}\text { Airway } \\
\text { protection: } \\
\mathrm{N}=28\end{array}$} \\
\hline & MRRH & MNRH & MRRH & MNRH & MRRH & $\mathrm{MNRH}$ & MRRH & MNRH \\
\hline & $\begin{array}{l}\mathrm{N}=27 \\
\mathrm{n}(\%)\end{array}$ & $\begin{array}{l}\mathrm{N}=31 \\
\mathrm{n}(\%)\end{array}$ & $\begin{array}{l}N=24, n \\
(\%)\end{array}$ & $\begin{array}{l}N=18, n \\
(\%)\end{array}$ & $\begin{array}{l}N=16, n \\
(\%)\end{array}$ & $\begin{array}{l}\mathrm{N}=20, \mathrm{n} \\
(\%)\end{array}$ & $\begin{array}{l}N=8, n \\
(\%)\end{array}$ & $\begin{array}{l}\mathrm{N}=20 \\
\mathrm{n}(\%)\end{array}$ \\
\hline $\begin{array}{l}\text { Gender- } \\
\text { specific }\end{array}$ & $\begin{array}{l}17 \\
(63.0)\end{array}$ & $\begin{array}{l}20 \\
(64.5)\end{array}$ & $15(62.5)$ & $11(61.1)$ & $\begin{array}{l}15 \\
(93.7)\end{array}$ & $\begin{array}{l}14 \\
(70.0)\end{array}$ & $\begin{array}{l}3 \\
(37.5)\end{array}$ & $\begin{array}{l}14 \\
(70.0)\end{array}$ \\
\hline Male & $\begin{array}{l}10 \\
(37.0)\end{array}$ & $\begin{array}{l}11 \\
(35.5)\end{array}$ & \multirow[t]{2}{*}{0.075} & \multirow[t]{2}{*}{0.584} & \multirow{2}{*}{$\begin{array}{l}1(6.3) \\
\mathbf{0 . 0 2 9}\end{array}$} & \multirow{2}{*}{$\begin{array}{l}6(30.0) \\
0.626\end{array}$} & $\begin{array}{l}5 \\
(62.5)\end{array}$ & $\begin{array}{l}6 \\
(30.0)\end{array}$ \\
\hline $\begin{array}{l}\text { Female } \\
p \text {-value }\end{array}$ & 0.054 & 0.777 & & & & & 0.010 & 0.626 \\
\hline $\begin{array}{l}\text { Age } \\
\text { category }\end{array}$ & $\begin{array}{l}3 \\
(11.1)\end{array}$ & $\begin{array}{l}8 \\
(25.8)\end{array}$ & $1(4.2)$ & $4(22.2)$ & $2(12.5)$ & $3(15.0)$ & $\begin{array}{l}1 \\
(12.5)\end{array}$ & $\begin{array}{l}3 \\
(15.0)\end{array}$ \\
\hline $\begin{array}{l}\text { Pediatric } \\
(<18)\end{array}$ & $\begin{array}{l}24 \\
(88.9)\end{array}$ & $\begin{array}{l}23 \\
(74.2)\end{array}$ & \multirow{2}{*}{$\begin{array}{l}23(95.8) \\
0.187\end{array}$} & $\begin{array}{l}14(77.8) \\
0.977\end{array}$ & \multirow{2}{*}{$\begin{array}{l}14 \\
(87.5) \\
0.686\end{array}$} & $\begin{array}{l}17 \\
(85.0)\end{array}$ & $\begin{array}{l}7 \\
(87.5)\end{array}$ & $\begin{array}{l}17 \\
(85.0)\end{array}$ \\
\hline $\begin{array}{l}\text { Adults } \\
(\geq 18) \\
\text { p-value }\end{array}$ & 0.777 & 0.407 & & & & $0.3<9$ & 0.797 & 0.329 \\
\hline
\end{tabular}

Tracheostomy-related complications

Overall, 53 patients developed complications, giving an incidence of early tracheostomy-related complications of 53\% (95\% Cl: $43.0-62.7$ ). No significant difference in incidence was noted between $\mathrm{MRRH}$ and $\mathrm{MNRH}, \mathrm{p}=0.841$.

A total of 76 complication events (38 in MRRH and 38 in MNRH) occurred among the 53 patients, of which 18 patients developed more than one complication. The commonest complications are; tube obstruction (52.6\%), inadvertent decannulation (17.1\%) and bleeding (11.8\%). Table 4

There was no significant difference in the distribution of the common tracheostomy-related complications between MRRH and MNRH except for inadvertent decannulation was commonest at MNRH $(26.3 \%)$ as compared to $7.9 \%$ at MNRH $(p=0.037)$. 
Table 4

Incidences of specific early complications, overall and by hospital stratification

\begin{tabular}{|lllll|}
\hline Complication & $\begin{array}{l}\text { Overall, } \mathbf{N}=\mathbf{7 6}, \\
\mathbf{n}(\%)\end{array}$ & $\begin{array}{l}\text { MNRH, N=38, } \\
\mathbf{n}(\%)\end{array}$ & $\begin{array}{l}\text { MRRH, N=38, } \\
\mathbf{n}(\%)\end{array}$ & p-value \\
\hline Tube Blockade & $40(52.6)$ & $20(52.6)$ & $20(52.6)$ & 1.000 \\
\hline Inadvertent decannulation & $13(17.1)$ & $10(26.3)$ & $3(7.9)$ & $\mathbf{0 . 0 3 7}$ \\
\hline Bleeding & $9(11.8)$ & $4(10.5)$ & $5(13.2)$ & 0.727 \\
\hline Stomal site Infection & $5(6.6)$ & $3(7.9)$ & $2(5.3)$ & 0.646 \\
\hline Subcutaneous emphysema & $3(3.9)$ & $0(0.0)$ & $3(7.9)$ & 0.079 \\
\hline Dysphagia & $3(3.9)$ & $1(2.6)$ & $2(5.3)$ & 0.558 \\
\hline Stomal granulation & $2(2.6)$ & $0(0.0)$ & $2(5.3)$ & 0.153 \\
\hline Aspiration & $1(1.3)$ & $0(0.0)$ & $1(2.6)$ & 0.315 \\
\hline
\end{tabular}

Factors associated with development of early tracheostomy-related complications

Table 5 shows the results of univariate and multivariate analysis for factors associated with tracheostomy related complications. 
Table 5

Results of univariate and multivariate analysis for factors associated with early tracheostomy-related complications.

\begin{tabular}{|c|c|c|c|c|c|}
\hline \multirow[t]{3}{*}{ Variable } & \multicolumn{3}{|c|}{ Univariate analysis } & \multicolumn{2}{|c|}{ Multivariate analysis } \\
\hline & \multirow{2}{*}{$\begin{array}{l}\text { Complication, } \\
\text { n (\%) }\end{array}$} & \multirow{2}{*}{$\begin{array}{l}\text { No } \\
\text { Complication, } n \\
(\%)\end{array}$} & \multirow{2}{*}{$\begin{array}{l}\text { Unadjusted } \\
\text { RR (95\% Cl) }\end{array}$} & Adjusted & \multirow{2}{*}{$\begin{array}{l}\mathrm{p}- \\
\text { value }\end{array}$} \\
\hline & & & & $\mathrm{RR}(95 \% \mathrm{Cl})$ & \\
\hline Hospital & $26(52.0)$ & $24(48.0)$ & 1.0 & & \\
\hline Mbarara & $27(54.0)$ & $23(46.0)$ & $1.1(0.72-$ & & \\
\hline \multicolumn{6}{|l|}{ Mulago } \\
\hline Gender & $12(40.0)$ & $18(60.0)$ & 1.0 & 1.0 & 0.132 \\
\hline Female & $41(58.6)$ & $29(41.4)$ & $\begin{array}{l}1.5(0.91- \\
2.36)\end{array}$ & $\begin{array}{l}1.4(0.91- \\
2.04)\end{array}$ & \\
\hline \multicolumn{6}{|l|}{ Male } \\
\hline Age category (Years) & $43(51.2))$ & $41(48.8)$ & 1.0 & 1.0 & 0.048 \\
\hline Adults ( $\geq 18)$ & $10(62.5)$ & $6(37.5)$ & $1.2(0.79-$ & $1.2(1.00-$ & \\
\hline \multicolumn{6}{|l|}{ Pediatric $(<18)$} \\
\hline \multirow{2}{*}{$\begin{array}{l}\text { Post-operative feeding } \\
\text { mode }\end{array}$} & $31(45.6)$ & $37(54.4)$ & 1.0 & & \\
\hline & $22(68.7)$ & $10(31.3)$ & $\begin{array}{l}1.5(1.06-13) \\
2.13\end{array}$ & & \\
\hline \multicolumn{6}{|l|}{ Oral Feeding } \\
\hline Admission Diagnosis & $23(67.7)$ & $11(32.3)$ & 1.0 & 1.0 & 0.234 \\
\hline \multirow{2}{*}{$\begin{array}{l}\text { Upper airway } \\
\text { obstruction }\end{array}$} & $28(46.7)$ & $32(53.3)$ & $0.7(0.48-$ & $0.8(0.57-$ & 0.317 \\
\hline & \multirow[t]{3}{*}{02 (33.3) } & \multirow[t]{3}{*}{$04(66.7)$} & & & \\
\hline TBI/stroke & & & $\begin{array}{l}0.5(0.15- \\
1.56)\end{array}$ & $\begin{array}{l}0.5(0.15- \\
1.84)\end{array}$ & \\
\hline Others & & & & & \\
\hline \multirow{2}{*}{$\begin{array}{l}\text { Post-operative } \\
\text { ventilation mode } \\
\text { Spontaneous }\end{array}$} & $19(41.3)$ & $27(58.7)$ & 1.0 & & \\
\hline & $34(63.0)$ & $20(37.0)$ & $\begin{array}{l}1.5(1.02-02 \\
2.27)\end{array}$ & & \\
\hline Ventilator assisted & & & & & \\
\hline
\end{tabular}

TBI: Traumatic brain injury, OMF: oromaxillofacial, ICU: intensive care unit, HDU: high dependency unit, ENT: ear, nose and throat 


\begin{tabular}{|c|c|c|c|c|c|}
\hline \multirow[t]{2}{*}{ Variable } & \multicolumn{3}{|c|}{ Univariate analysis } & \multicolumn{2}{|c|}{ Multivariate analysis } \\
\hline & $\begin{array}{l}\text { Complication, } \\
\text { n (\%) }\end{array}$ & $\begin{array}{l}\text { No } \\
\text { Complication, } n \\
\text { (\%) }\end{array}$ & $\begin{array}{l}\text { Unadjusted } \\
\text { RR (95\% Cl) }\end{array}$ & $\begin{array}{l}\text { Adjusted } \\
\text { RR (95\% Cl) }\end{array}$ & $\begin{array}{l}\mathrm{p} \text { - } \\
\text { value }\end{array}$ \\
\hline Site for surgery & $7(50.0)$ & $7(50.0)$ & 1.0 & 1.0 & 0.609 \\
\hline Wards (ENT/OMF) & $4(57.2)$ & $3(42.8)$ & $1.4(0.49-$ & $1.2(0.55-$ & 0.083 \\
\hline Emergency/casualty & $25(46.3)$ & $29(53.7)$ & & & 0.127 \\
\hline ICU/HDU & $17(68.0)$ & $8(32.0)$ & $\begin{array}{l}0.98) \\
1.68)\end{array}$ & $2.79)$ & \\
\hline Operating theatre & & & $\begin{array}{l}1.4(0.75- \\
2.45)\end{array}$ & $\begin{array}{l}1.5(0.88- \\
2.64)\end{array}$ & \\
\hline Indication for & $29(50.0)$ & $29(50.0)$ & 1.0 & 1.0 & 0.005 \\
\hline Pulmonary toilet & $16(38.1)$ & $26(61.9)$ & $\begin{array}{l}1.7(1.08- \\
2.58)\end{array}$ & $\begin{array}{l}1.8(1.19- \\
2.76)\end{array}$ & 0.605 \\
\hline $\begin{array}{l}\text { Anticipated prolonged } \\
\text { intubation }\end{array}$ & $\begin{array}{l}16(57.1) \\
5(55.6)\end{array}$ & $\begin{array}{l}12(42.9) \\
4(44.4)\end{array}$ & $\begin{array}{l}1.1(0.75- \\
1.64)\end{array}$ & $\begin{array}{l}1.4(0.79- \\
1.64)\end{array}$ & $\begin{array}{l}0.872 \\
0.062\end{array}$ \\
\hline Airway protection & $24(66.7)$ & $12(33.3)$ & $\begin{array}{l}1.1(0.57- \\
1.95)\end{array}$ & $\begin{array}{l}1.3(0.57- \\
1.90)\end{array}$ & \\
\hline $\begin{array}{l}\text { Anticipated difficult } \\
\text { intubation } \\
\\
\text { Upper airway } \\
\text { obstruction }\end{array}$ & & & $\begin{array}{l}1.5(1.03- \\
2.09)\end{array}$ & $\begin{array}{l}1.5(1.03- \\
2.09)\end{array}$ & \\
\hline Tracheostomy type & $28(49.1)$ & $29(50.1)$ & 1.0 & & \\
\hline $\begin{array}{l}\text { Elective } \\
\text { Emergency }\end{array}$ & $25(58.0)$ & $18(42.0)$ & $\begin{array}{l}1.2(0.82- \\
1.70)\end{array}$ & & \\
\hline Type of tracheal & $17(43.6)$ & $22(56.4)$ & 1.0 & 1.0 & 0.016 \\
\hline T-Incision & $8(88.9)$ & $1(11.1)$ & $\begin{array}{l}2.0(1.33- \\
3.11)\end{array}$ & $\begin{array}{l}1.6(1.09- \\
2.43)\end{array}$ & 0.037 \\
\hline & $26(54.2)$ & $22(45.8)$ & & & 0.165 \\
\hline $\begin{array}{l}\text { Bjork Flap } \\
\text { Vertical }\end{array}$ & $2(50.0)$ & $2(50.0)$ & $\begin{array}{l}1.2(0.79- \\
1.93)\end{array}$ & $\begin{array}{l}1.5(1.02- \\
2.27)\end{array}$ & \\
\hline Horizontal & & & $\begin{array}{l}1.1(0.40- \\
3.25)\end{array}$ & $\begin{array}{l}1.6(0.83- \\
2.99)\end{array}$ & \\
\hline
\end{tabular}

TBI: Traumatic brain injury, OMF: oromaxillofacial, ICU: intensive care unit, HDU: high dependency unit, ENT: ear, nose and throat 


\begin{tabular}{|c|c|c|c|c|c|}
\hline \multirow[t]{3}{*}{ Variable } & \multicolumn{3}{|c|}{ Univariate analysis } & \multicolumn{2}{|c|}{ Multivariate analysis } \\
\hline & Complication, & & Unadjusted & Adjusted & p- \\
\hline & & $\begin{array}{l}\text { Complication, } \mathrm{n} \\
\text { (\%) }\end{array}$ & $\mathrm{RR}(95 \% \mathrm{Cl})$ & $\mathrm{RR}(95 \% \mathrm{Cl})$ & \\
\hline $\begin{array}{l}\text { Cuffed tracheostomy } \\
\text { tube }\end{array}$ & $45(50.6)$ & $44(49.4)$ & 1.0 & & \\
\hline Yes & $8(72.7)$ & $3(27.3)$ & $\begin{array}{l}1.4(0.94- \\
2.18)\end{array}$ & & \\
\hline \multicolumn{6}{|l|}{ No } \\
\hline \multirow{2}{*}{$\begin{array}{l}\text { Peri-operative } \\
\text { antibiotics }\end{array}$} & $27(45.0)$ & $33(55.0)$ & 1.0 & & \\
\hline & $26(65.0)$ & $14(35.0)$ & $\begin{array}{l}1.4(1.00- \\
2.07)\end{array}$ & & \\
\hline \multicolumn{6}{|l|}{ No } \\
\hline $\begin{array}{l}\text { TBI: Traumatic brain in } \\
\text { unit, ENT: ear, nose an }\end{array}$ & $\begin{array}{l}\text { MFF: oromaxi } \\
\text { at }\end{array}$ & cial, ICU: inten & care unit, $\mathrm{H}$ & high depen & \\
\hline
\end{tabular}

In univariate analysis, the statistically significant factors were; Oral Feeding post-operatively, assisted post-operative ventilation, having an indication of anticipated prolonged intubation or upper airway obstruction, use of Bjork flap tracheal incision and peri-operative antibiotic use. In addition to these factors, admission diagnosis, age, gender, and the site for surgery were included in the final model and adjusted for.

In multivariate analysis, the only factors with a significant independent association with early tracheostomy-related complications were; being younger than 18years, having an indication for tracheostomy of anticipated prolonged intubation, and having either a Bjork flap or a vertical tracheal incision.

Patients having anticipated prolonged intubation as the indication for tracheostomy had a 1.8 times risk of developing early complications compared to patients with pulmonary toilet as their indication ( $R R=1.8$, $95 \% \mathrm{Cl}: 1.19-2.76, \mathrm{p}=0.005)$. Patients who had a Bjork flap tracheal incision had a 1.6 times risk of developing early complications ( $R R=1.6,95 \% \mathrm{Cl}: 1.09-2.43, p=0.016)$ while those with vertical incision had a 1.5 times risk compared to those that received a T-incision ( $R R=1.53,95 \% \mathrm{Cl}: 1.02-2.27, p=0.037)$. Patients aged below 18 years had a 1.2 times risk of early complications as compared to those 18 years and above (RR=1.22, 95\% Cl: $1.00-1.47, p=0.048)$.

\section{Discussion}

\section{Indications for tracheostomy}

In this study, we observed that a considerable proportion (69\%) of patients had more than one tracheostomy indication. This finding was probably because most (54\%) of study participants were very 
ill ICU and HDU patients who are likely to have multiple challenges for example; reduced consciousness hence unable to protect their airways and clear airway secretions yet at the same time require prolonged assisted intubation, all of which are recognized tracheostomy indications. This study found a comparable distribution of indications at the two study sites probably because of the similarity in patients' sociodemographic characteristics as shown in Table 1, or disease epidemiology and risk for trauma given that they are both urban-based hospitals. We also found pulmonary toilet as the commonest indication which is at variance with what was previously reported in various African studies that found upper airway obstruction as the commonest indication (Adetinuola, Amusa et al. 2011, Ammar, Haider Wahab et al. 2012, Adedeji T, Olaosun OA et al. 2014, B. Alabi, O. Afolabi et al. 2018) while others reported anticipated prolonged intubation as the commonest at $55.2 \%$ and $95 \%$ in Rwanda and India respectively (Charles and Mukara 2018, Chavan, Ingole et al. 2019). The discrepancy may have been because comparison studies that found upper airway obstruction as commonest differed from ours in regard to admission diagnoses. They reported significant contributions from aero-digestive infections and impacted airway foreign bodies among children, and aerodigestive trauma and tumors especially among their adult patients all of which contributed to the rampant upper air-way obstruction. Our study also had few pediatric patients (16\%) and with no patients with aero-digestive infections or airway foreign bodies. Overall, the commonest form of trauma we found was traumatic brain injury rather than aerodigestive trauma reported in other studies. In the same vein, the Indian study had $59 \%$ and $21.3 \%$ of their patients admitted due to severe organophosphate poisoning and snake bites respectively while in Rwanda, all their patients were recruited from ICU and had history of severe trauma, all of whom were likely to require prolonged periods of intubation for airway support. The present study however did not find similar indications and the trauma severity was not assessed.

\section{Early tracheostomy-related complications}

The $53 \%$ incidence of early tracheostomy-related complications documented in this study, although in alignment with the global estimated range of 6 to $66 \%$, is higher than the $21.5 \%$ reported in Tanzania (Gilyoma, Balumuka et al. 2011). Similarly, this incidence is higher than what has been reported in previous studies from comparable settings such as Nigeria where this rate ranged between $10.3 \%$ and 21\% (Adetinuola, Amusa et al. 2011, Adedeji T, Olaosun OA et al. 2014) and India (29.8\%) (Kawale, Keche et al. 2017). Lower complication rates (15\%) have been documented in high income settings such as Finland (Ruohoalho, Xin et al. 2021). Notably most of these studies were retrospective and considered all post-procedure complications and not only early complications. The high incidence in our study could be attributed to the cadre of surgeons performing the procedure in our case who were mainly residents in training unlike similar studies where mainly specialist otolaryngologists with arguably better skills and longer experience performed the procedures (Ruohoalho, Xin et al. 2021). The observational nature of this study too could have triggered an increased intentional surveillance for complications resulting in the documentation of even more subtle episodes of tube obstruction hence providing a higher incidence as also seen in Kenya (Karuga, Oburra et al. 2012).

\section{Incidence of specific early complications}

Page $14 / 19$ 
Our study reported tracheostomy tube obstruction as the commonest early complication (52.6\%) which agrees with another study in similar settings that reported $80.3 \%$ of patients in Kenya (Nyansikera and Kirui 2013). Our finding could be due to the fact that majority (72\%) of tubes used had no disposable internal cannula which are known to ease tube care, in addition to absence of heat and moisture exchangers as well as the low staffing, a challenge that was similarly highlighted by the Nyansikera and Kirui study. This makes it harder to achieve the minimum of 3 times per day tracheostomy tube suctioning as recommended (Morris, Whitmer et al. 2013) to prevent this complication. Life-threatening complications like tracheo-innominate fistula, pneumothorax and pneumomediastinum were not reported in this study and no mortality occurred as a result of tracheostomy.

\section{Factors associated with development of early tracheostomy-related complications}

Bearing in mind tracheostomy tube obstruction as the commonest complication in this study, our finding that anticipated prolonged intubation as an indication increased the risk of early complications 1.8 times compared to pulmonary toilet may be because patients who were operated for this indication were more likely to have had a significantly severe illness. Such patients would still be too sick to self-care for their tracheostomy tubes even in the post-procedure period and this may be compounded by their inability to communicate a need to be suctioned or have the tube cleaned or changed in addition to a higher possibility of a poor cough reflex. All these may have increased chances of retention of copious airway secretions hence a higher risk of tube obstruction. Such patients are also more likely to require assisted bed turning and bathing compared to more stable patients and this increases chances of inadvertent tube decannulation, which was in fact the second most common complication $(17.1 \%)$ in this study.

Our finding that patients younger than 18years had an increased risk of early complications was not surprising since this association had previously been reported by a study in a similar setting that found a higher complication rate among children below 10years as compared to older patients (Gilyoma, Balumuka et al. 2011). This is in agreement with our study in which 10 out of the 16 children enrolled were below 10 years of age. This age group could be more prone to complications because of their anatomical features such as a shorter neck, more pliable laryngeal structures and more prominent subcutaneous fat. Also, pediatric tracheostomy requires procedural modifications if complications are to be minimized. For example, neck hyperextension may pull mediastinal structures in to the neck thus increasing chances of damage to lung apices and resultant emphysema, pneumothorax and pneumomediastinum. Also, awake pediatric patients are still less likely to communicate the need to be suctioned which increases chances of tube obstruction as compared to adults.

The Bjork flap is an inferiorly based tracheal flap through the $2 \mathrm{nd}, 3 \mathrm{rd}$, and 4th tracheal rings which is fixed to the skin to stabilize the tracheal lumen. Although it has a risk for tracheal stenosis, it has advantages over traditional incisional and excisional window procedures including; reduced risk of false cannulation especially during emergency recannulation following accidental tube dislodgement, early stomal maturation and ease of stomal care by assistants and family members (Au, Heineman et al. 2017, Singh, Goyal et al. 2017, Mukherjee, Samaddar et al. 2020). These make it the preferred tracheal incision 
in our setting for patients who require tracheostomy for longer durations such as those with head and neck tumors. However, its indications may vary, as it was preferred for patients with prolonged mechanical ventilation and elevated body mass index while window procedures were used in head and neck cancer patients in USA (Kennedy, Abdel-Aty et al. 2021).

Our finding that Bjork flap and vertical tracheal incisions were associated with increased risk of early complications and the fact that tracheostomy tube obstruction was the commonest complication across all tracheal incisions including patients with Bjork flap (7 out of 9) cannot be conclusively explained by this study. There is need to further explore this relationship among patients who receive Bjork flap.

The strength of our study is that it gives information on tracheostomy-related complications in Uganda within a period preceding the COVID-19 pandemic and so could be used as baseline assessment for future studies that will assess the impact of COVID-19 pandemic on tracheostomy outcomes. However, since the study was done in the pre-COVID-19 period, its findings may need to be interpreted with caution within the current COVID-19 context. Also, the small sample size may have limited its ability to establish true associations between complications and the risk factors identified. Furthermore, the small number of pediatric patients in this study may affect the generalizability of our study results to the pediatric age group.

\section{Conclusion And Recommendation}

Within a pre-COVID-19 context, pulmonary toilet is the commonest tracheostomy indication at major hospitals in Uganda. The incidence of early tracheostomy complications is high and majorly related to post-procedure tracheostomy tube management. Having anticipated prolonged intubation as an indication for tracheostomy, a Bjork flap or vertical tracheal incisions and being a child increases the risk of complications. Emphasis on surgical training and post-procedural management for both clinicians and nurses should be made. Future studies will be needed for better understanding about the possible impact of the COVID-19 pandemic on the outcomes of tracheostomy in the same context.

\section{Declarations}

\section{Ethical approval:}

Clearance from the MUST Faculty of Medicine Research Committee (FREC), and Mbarara University Research Ethics Committee (MUREC number 01/11-17).

\section{Consent for publication:}

Not applicable

\section{Availability of data and materials:}


The datasets used and/or analyzed during the current study available from the corresponding author on reasonable request.

\section{Competing interests:}

The authors declare that they have no competing interests.

\section{Funding:}

Self-funded

\section{Author's contributions:}

DJN, DA and DN conceived the idea. DJN, CAN and VN collected the data and assessed the patients. DJN, DN and DA analyzed the data. All authors reviewed the tables of results. DJN wrote the first draft of the manuscript. All authors reviewed and approved the final draft of manuscript

\section{Acknowledgements:}

Dr. Justine Namwagala offered guidance throught the study. Drs. Ruth Kagere and Abdirahman Jamaa assisted in patient recruitment and data collection, All residents in the MNRH and MRRH Otolaryngology clinics participated in the overall care for study participants including indentification and management of complications.

Institution of affiliation where the study was conducted: Mbarara University of Science and Technology, Faculty of Medicine, Department of Otolaryngology, Uganda.

\section{References}

1. Abe T, et al. "Epidemiology and patterns of tracheostomy practice in patients with acute respiratory distress syndrome in ICUs across 50 countries.". Crit Care. 2018;22(1):1-16.

2. Adedeji T, et al. "Indications and outcomes of tracheostomy: An experience in a resource-limited environment..". HJHealth Res Rev. 2014;1:40-3.

3. Adetinuola, et al. "Tracheostomy in south western Nigeria: Any change in pattern?". Journal of medicine medical science. 2011;2(7):997-1002.

4. Ammar, et al. "Tracheostomy: Cross sectional study of indications in relation to age group in alyarmouk teaching hospital. " Basrah Journal Of Surgery; 2012.

5. Au JK, et al. "Should adult surgical tracheostomies include a Bjork flap?". Laryngoscope. 2017;127(3):535-6.

6. Alabi B, et al. Indications and outcomeof tracheostomy in Ilorin, North central Nigeria: 10 years review. Annals of African Medicine. 2018;17(1):1-6. 
7. Barry and Bodenham. The role of tracheostomy in ICU. Anaesthesia Intensive Care Medicine. 2004;5(11):375-8.

8. Brenner MJ, et al. Global Tracheostomy Collaborative: data-driven improvements in patient safety through multidisciplinary teamwork, standardisation, education, and patient partnership. $\mathrm{Br} \mathrm{J}$ Anaesth. 2020;125(1):e104-18.

9. Charles N, Mukara KB. "Indications and Immediate Outcomes of Tracheostomy in Rwanda ". Annals of African Surgery. 2018;15(2):52-6.

10. Chavan RP, et al. (2019). "Tracheostomy: Experience at Tertiary Hospital." Indian J Otolaryngol Head Neck Surg 71.

11. Cheung and Napolitano. (2014). "Tracheostomy: Epidemiology, Indications, Timing, Technique, and Outcomes." Respiratory Care 59(6).

12. Cramer JD, et al. "Mortality associated with tracheostomy complications in the United States: 20072016.". Laryngoscope. 2019;129(3):619-26.

13. Gilyoma, et al. (2011). "Ten-year experiences with Tracheostomy at a University teaching hospital in Northwestern Tanzania: A retrospective review of 214 cases." World Journal of Emergency Surgery $6(38)$.

14. Halum, et al. "A multi-institutional analysis of tracheotomy complications.". Laryngoscope. 2012;122(1):38-45.

15. Hamid, et al. (2011). "Complications of tracheostomy." Journal of Postgraduate Medical Institute (Peshawar-Pakistan) 18(3).

16. Johnson, et al. Bailey's Head and Neck Surgery - otolaryngology. Philadelphia Lippinkott Williams and Wilkins; 2014.

17. Karuga, et al. "Risk Factors of Early Complications of Tracheostomy at Kenyatta National Hospital." East and Central African. Journal of Surgery. 2012;17(1):51-6.

18. Kawale MA, et al. (2017). "A Prospective Study of Complications of Tracheostomy and Management in Tertiary Care Hospital in Rural Area." Global Journal of Otolaryngology - Head and neck Surgery $5(3)$.

19. Kawale MA, et al. A prospective study of complications of tracheostomy and management in tertiary care hospital in rural area. Int J Otorhinolaryngol Head Neck Surg. 2017;3:687-92.

20. Kelsey, et al. Methods of sampling and estimation of sample size. New York: Oxford University Press; 1996.

21. Kennedy MM, et al. Comparing tracheostomy techniques: Bjork flap vs. tracheal window. Am J Otolaryngol. 2021;42(6):103030.

22. Morris LL, et al. Tracheostomy care and complications in the intensive care unit. Critical care nurse. 2013;33(5):18-30.

23. Mukherjee $D$, et al. "Conventional versus flap tracheostomy: operative simplicity, stomal care, complications.". Bengal Journal of Otolaryngology Head Neck Surgery. 2020;28(2):120-6. 
24. Nyansikera and Kirui. (2013). Quality of tracheostomy care: A case of kenyatta national hospital, nairobi county. Nursing, University of Nairobi. Master of science (medical surgical nursing).

25. Ruohoalho J, et al. (2021). "Tracheostomy complications in otorhinolaryngology are rare despite the critical airway." European Archives of Oto-Rhino-Laryngology: 1-5.

26. Saleem SM. (2018). "modified kuppuswamy scale updated for year 2018." paripex - Indian journal of research 7(3).

27. Singh R, et al. "Bjork Flap" J Otolaryngol ENT Res. 2017;7(1):00192.

28. Taylor and Randal. Open Tracheostomy Procedure. Atlas Oral Maxillofacial Surgery. Elsevier Inc; 2015. 